\title{
Trauma torácico: análise de 124 pacientes submetidos à toracotomia
}

\section{Thoracic trauma: analysis of 124 patients who underwent thoracotomy}

Fernando luiz Westphal, tCBC-AM¹; Luiz Carlos de Lima²; José Correa lima Netto33; Jeancarllo de Souza da Silva ${ }^{4}$; Vítor Lazarini dos Santos Júnior ${ }^{5}$; Danielle CRIStine Westphal ${ }^{6}$

\section{RE S U M O}

\begin{abstract}
Objetivo: Analisar os pacientes submetidos à toracotomia para o tratamento de traumatismo torácico. Métodos: Estudo retrospectivo por meio da análise de prontuários nos dois principais hospitais de referência para trauma em adultos desta cidade, por um período de cinco anos, interessando dados epidemiológicos, agente causal, indicações, tipo de incisão, classificação do escore anatômico do trauma, fatores prognósticos e a mortalidade. Resultados: Foi analisado neste estudo 124 pacientes submetidos à toracotomia com idade média de 28 anos, tendo como o agente causal mais incidente a arma branca (68\% dos casos). A principal indicação da toracotomia foi hemotórax maciço com $50,7 \%$ dos casos, seguido de choque cardiogênico ou hipovolêmico com $48,4 \%$. Ocorreram 28 óbitos (20,6\%), sendo que os pacientes com lesões de veia cava (cinco pacientes) e aorta (dois pacientes) tiveram uma mortalidade de $100 \%$. Observou-se uma maior mortalidade em pacientes com escore anatômico do trauma superior a $14(p=0,004)$ e maior quantidade de sangue transfundido $(p=0,090)$. Conclusão: O perfil do paciente que foi vítima de traumatismo torácico e submetido à toracotomia exploradora é o seguinte: jovem, do sexo masculino e vítima de trauma por arma branca. Os fatores que mais contribuíram para o êxito letal foram o elevado escore anatômico do trauma e a associação com lesões vasculares importantes, como da artéria aorta e veia cava.
\end{abstract}

Descritores: Traumatismos torácicos. Toracotomia. Índices de gravidade do trauma.

\section{INTRODUÇÃO}

O trauma é uma das principais causas de morte na atualidade, ocasionado pelo aumento da violência urbana e pelo avanço tecnológico da indústria automotiva, que fabrica veículos e motos mais potentes, com uma maior propensão a desencadear acidentes graves. Seguindo a tendência nacional, a Região Norte de nosso país experimenta um aumento do número de pacientes acometidos pelo trauma, tanto causado por acidentes quanto por agressões físicas. Há um grande contingente de pessoas que está exposta à violência, principalmente na periferia das grandes cidades, onde o desemprego e a utilização de drogas são mais freqüentes'.

A maioria dos traumatismos torácicos é tratada prontamente com a drenagem pleural e somente cerca de $15-30 \%$ destes necessitarão de uma toracotomia para a resolução das injúrias intratorácicas².

As indicações para a toracotomia nas primeiras horas de admissão nos prontos-socorros geralmente estão relacionadas a choque hipovolêmico, tamponamento cardíaco ou insuficiência respiratória por intenso escape aéreo pelo dreno torácico. Nesta situação, os órgãos mais acometidos dentro da cavidade torácica são os pulmões, o coração, os grandes vasos e as vias aéreas maiores. Passado este primeiro momento, as toracotomias são realizadas para o tratamento de coágulo retido, perfurações de esôfago torácico ou para tratamento de processo infeccioso pleura ${ }^{1-3}$.

O objetivo deste trabalho é analisar os pacientes submetidos à toracotomia para o tratamento de traumatismo torácico, em Hospitais de Referência para Trauma, na cidade de Manaus.

\section{MÉTODOS}

Estudo retrospectivo de pacientes vítimas de trauma torácico submetidos a tratamento por toracotomia nos Prontos-Socorros 28 de Agosto e Dr. João Lúcio Pereira Machado, na cidade de Manaus, no período entre janeiro

Trabalho realizado no Hospital Pronto-Socorro Municipal 28 de Agosto e no Hospital Pronto-Socorro João Lúcio Pereira Machado Manaus - AMBR.

1. Professor Doutor da Disciplina de Cirurgia Torácica da Universidade da Universidade Federal do Amazonas, Manaus, AM-BR. 2. Professor Doutor da Disciplina de Cirurgia Torácica da Universidade da Universidade Federal do Amazonas, Manaus, AM-BR. 3.Especialista em Cirurgia Torácica da Universidade Federal do Amazonas, Manaus, AM-BR. 4.Especialista em Cirurgia Geral da Universidade Federal do Amazonas, Manaus, AM-BR. 5. Acadêmico de Medicina da Universidade do Estado do Amazonas, Manaus, AM-BR. 6. Acadêmica de Medicina da Universidade Federal do Amazonas, Manaus, AM-BR. 
de 1997 a dezembro de 2001. Este trabalho foi aprovado pelo Comitê de Ética em Pesquisa da Universidade Federal do Amazonas.

As seguintes variáveis foram analisadas: perfil epidemiológico, condições de admissão no hospital, mecanismo de trauma, órgão torácico acometido, trauma extratorácico associado, local da toracotomia, via de acesso, tipo de operação realizada, número de concentrado de hemácias utilizado e mortalidade.

Os critérios de inclusão foram: a presença de sinais vitais à admissão na sala de operação e pacientes submetidos à toracotomia para o tratamento de trauma nos dois prontos-socorros previamente selecionados. Foram excluídos os pacientes que não possuíam dados, em prontuário, suficientes para o preenchimento do protocolo.

A classificação da gravidade do trauma foi realizada por meio do Escore Anatômico de Trauma (ISS - Injury Severity Score) que leva em consideração a região anatômica atingida e o grau de gravidade em cada região, variando entre 1 e 5, elevando-se ao quadrado a pior lesão dos três segmentos corporais mais acometidos, classificando o trauma em leve $(<9)$, moderado (9 a 14) e grave $(>14)$.

A análise estatística dos dados foi realizada por meio do uso do programa EPI INFO 6.04 (CDC - Centers for Disease Control \& Prevention, USA), com o cruzamento e comparação entre os grupos estudados. Nas análises estatísticas usou-se o nível alfa de 5\% $(p=0,05)$. A análise dos dados entre as variáveis foi realizada pela aplicação do teste do qui-quadrado.

\section{RESULTADOS}

Este estudo analisou 124 pacientes com a idade média de 28 anos e houve predominância do sexo mascu- lino com 96,8\% dos casos. Observou-se um alto índice de acometimento dos jovens no trauma, com 89,5\% dos casos incluídos até a quarta década. O agente causal mais frequente foi a arma branca com 69,1\%, seguidos por arma de fogo $(21,4 \%)$, trauma contuso $(8,7 \%)$ e outros $(0,8 \%)$.

A toracotomia foi indicada nos casos de choque cardiogênico ou hipovolêmico em $48,4 \%$ dos casos, o hemotórax volumoso em 50,7\%. Toracotomia traumática e lesão de vias aéreas corresponderam cada a 0,8\% das indicações de tratamento cirúrgico por toracotomia.

As vias de acesso mais utilizadas (Figura 1) foram a toracotomia esquerda (47\%), independente de ser anterior ou anterolateral, seguidas por toracotomia direita (20\%), bitoracotomia anterior (16\%) e esternotomia (9,5\%).

Observou-se uma frequência maior do trauma exclusivamente torácico (61\%) em relação ao trauma com lesões associadas (39\%). Dentre os órgãos intratorácicos, as lesões pulmonares foram as mais frequentes (38\%), com predomínio no pulmão esquerdo (52,7\%). Seguido destas, estão as lesões cardíacas (30\%), com acometimento mais frequentes dos ventrículos direito e esquerdo com 30\% cada. As lesões vasculares vêm em seguida com 36 casos $(28,6 \%)$. As lesões vasculares mais encontradas foram de veias e artérias torácicas internas (26\%), seguidas por lesões de vasos musculares (19\%), artérias intercostais, veia cava inferior, veia cava superior, aorta torácica e vasos pulmonares.

As principais estruturas abdominais associadas ao trauma foram o diafragma, o fígado e o baço. As complicações foram observadas em $22,5 \%$ dos pacientes, sendo que as mais comuns foram hemotórax coagulado em nove pacientes, febre e empiema pleural em sete.

Em relação à mortalidade dos pacientes vítimas de trauma torácico submetidos à toracotomia, ocorreram vinte e seis óbitos, correspondentes a uma taxa de 20,6\% de mortalidade.

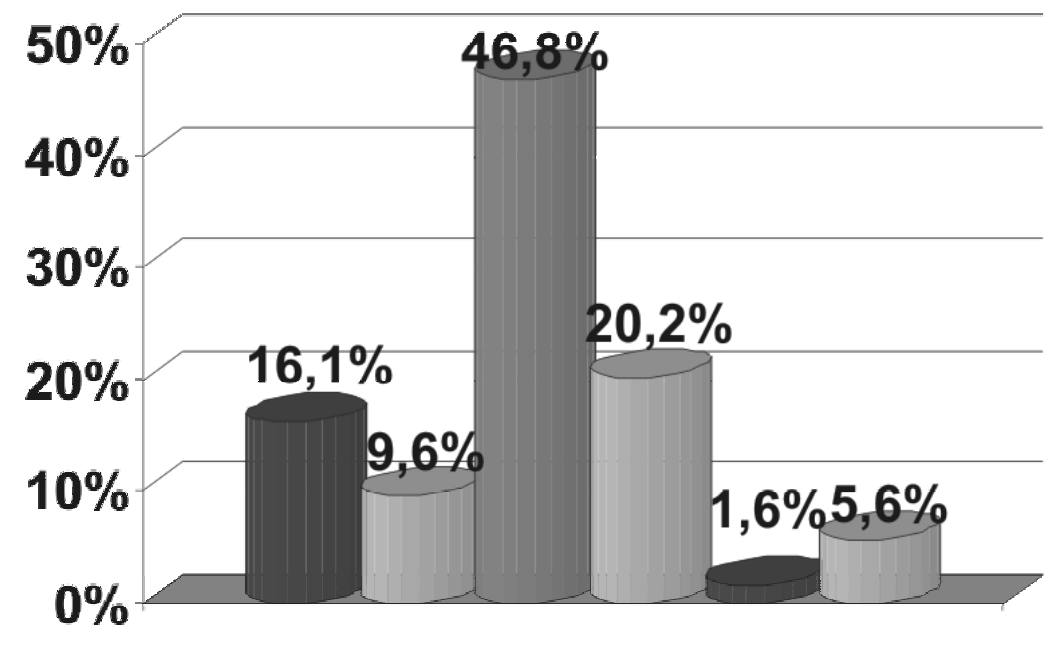

\begin{tabular}{|l|}
\hline Bitoracotomia \\
Esternotomia \\
Toracotomia E \\
Toracotomia D \\
Toracofreno- \\
laparotomia \\
Outras
\end{tabular}

Figura 1 - Distribuição dos pacientes pelo tipo de incisão cirúrgica realizada. 
Em relação à gravidade das lesões, o escore anatômico de trauma (Figura 2) apresentou uma proporção semelhante de pacientes com lesões graves (41,3\%) e leves (48,4\%). Entretanto, percebe-se uma maior incidência de óbitos em pacientes com escores graves (superiores a 14) em relação aos demais pacientes, apresentando-se com significância estatística $(p=0,004)$. Observou-se uma relação direta entre o agente causal e a mortalidade, pois os traumas contusos evoluíram mais a óbito proporcionalmente que as feridas por arma branca.

Quanto à utilização de concentrado de hemácias, observou-se uma tendência entre a mortalidade e a utilização de quatro ou mais unidades, indicando um pior prognóstico para estes pacientes $(p=0,09)$.

Nos traumas torácicos com comprometimento de órgãos nobres como coração, pulmões e vasos importantes houve uma menor taxa de sobrevida nos pacientes acometidos por lesões de grandes vasos (66\%), quando comparados com as lesões cardíacas ( $75 \%$ ) ou pulmonares $(78,2 \%)$. A mortalidade nas lesões vasculares da veia cava inferior (cinco casos) e da aorta torácica (três casos) foi de 100\%.

\section{DISCUSSÃO}

A etiologia do trauma torácico está relacionada às condições de desenvolvimento da população em estudo. No Brasil, a maioria dos pacientes atendidos nas emergências são consequências da violência urbana, com predominância das agressões físicas e acidentes automobilísticos. Assim, existe uma prevalência dos ferimentos penetrantes causados por arma branca ou de fogo, fato também observado em outros países latino-americanos, tais como em Cuba e no Chile, ${ }^{4,5}$. Diferentemente, em alguns países desenvolvidos, com um Índice de Desenvolvimento Humano alto, há uma escassez destes pacientes, influenci- ando negativamente na formação do cirurgião geral como referido recentemente por Bergeron et ${ }^{\prime}{ }^{6}$, do Canadá, no qual os residentes têm pouco treinamento em traumas penetrantes durante a formação.

O trauma surge na grande maioria das vezes pela agressão física, acometendo principalmente indivíduos do sexo masculino $98,8 \%$ em nosso estudo. Isto ocorre geralmente por disputas entre grupos de rua ou em bares, mais frequentados por indivíduos do sexo masculino. Este achado é semelhante ao de Spencer Netto et al (2000), em São Paulo, num estudo de traumatismo cardíaco, revelaram a taxa de $94,3 \%$ de pacientes do sexo masculino ${ }^{3}$, assim como em Alagoas?.

No presente estudo, foi encontrada uma alta incidência dos jovens como vítima do traumatismo torácico, dos quais 46,8\% estão entre 20 e 29 anos, em um intervalo de 15 a 78 anos. Entretanto, quando analisamos a faixa

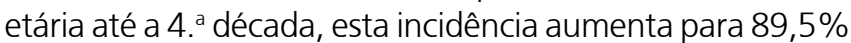
dos casos. Essa tendência no acometimento em adultos jovens é observada em outros locais, como em Cuba, com acometimento de $54,5 \%$ das vitimas de trauma nessa faixa etária4.

Nos ferimentos penetrantes há uma relação entre o tipo de arma utilizada para a agressão e as condições econômicas da população em estudo, pois nas regiões mais pobres há uma maior frequência na utilização de arma bran$\mathrm{ca}$, visto que tem um custo menor que a arma de fogo. Na nossa amostra observa-se esta relação, como relatado em outras séries ${ }^{8}$.

Na Região Norte e no Nordeste há uma nítida predominância da incidência de ferimentos por arma branca, como demonstrado neste e em outros trabalhos ${ }^{7,8}$. A frequência dos agentes etiológicos envolvidos na gênese dessa doença, foi comparável ao estudo de Fontelles e Mantovani et al (2000), que cita o envolvimento da arma branca $(59,7 \%)$, arma de fogo $(25 \%)$, outras causas $(9,7 \%)$

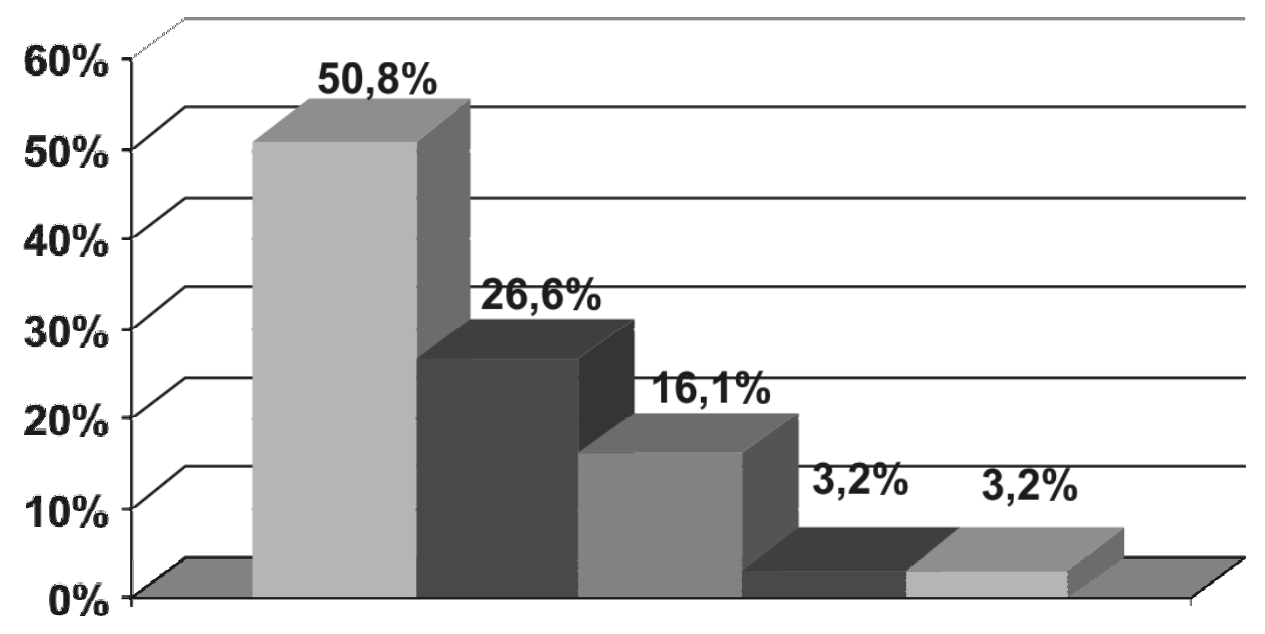

$\square 1$ a $10 \square 11$ a $20 \square 21$ a $30 \square 31$ a $40 \square>40$

Figura 2 - Distribuição dos pacientes quanto ao valor do Escore Anatômico de Trauma (Injury Severety Score / ISS). 
e contusão (7,3\%) na ordem decrescente de frequência. Os resultados do presente estudo foram semelhantes: em relação à arma branca em $69,1 \%$ dos casos, à arma de fogo em $21,4 \%$, à contusão em $8,7 \%$ e a outras causas em $0,8 \%$. A maior incidência de pacientes com trauma penetrante em relação ao contuso pode ser explicada pela característica da amostra que é formada por pacientes submetidos à toracotomia, que tem indicação mais restrita nos casos de trauma contuso ${ }^{9}$.

A associação do trauma torácico com o acometimento de outras partes do corpo pode ser uma forma de avaliação da gravidade do trauma. Entretanto, percebe-se o predomínio de lesões exclusivamente torácicas em relação às lesões associadas com outras áreas corporais em 61,1 e 38,9\%, respectivamente. Segundo Lima et al (1986), em um estudo retrospectivo com pacientes vítimas de trauma torácico, houve associação de traumatismos extratorácicos em 31\% dos pacientes, corroborando os dados relatados nesse estudo. Esta maior incidência de trauma torácico isolado, provavelmente, está relacionada ao agente causal mais frequente, a arma branca, pois nos traumas contusos a freqüência de associação com outros sistemas é muito mais elevada?.

Lima et al (1986) encontraram a lesão do parênquima pulmonar como a mais frequente $(36,6 \%)$, seguida por lesões de parede (24,4\%), grandes vasos $(2,8 \%)$ e cardíacas (2,1\%). No presente estudo, o órgão mais acometido foi o pulmão (43\%), seguido por lesões cardíacas (34\%), vasculares (31\%) e outras $(6,3 \%)$. Os achados entre os dois estudos foram diferentes porque esta casuística foi direcionada para pacientes submetidos à toracotomia exploradora, diferentemente do outro estudo, que abordou os pacientes atendidos como trauma torácico, mas nem todos foram submetidos à toracotomia? .

Ao avaliar lesões específicas de cada órgão, observa-se que os pulmões foram os mais acometidos, com ligeiro predomínio do pulmão esquerdo (52,7\%), em contraste com o trabalho de Spencer Netto et al (2000), citam o envolvimento pulmonar em $69 \%$ dos pacientes, onde o pulmão direito estava envolvido em $28 \%$ dos casos, e o esquerdo em $71 \%{ }^{3}$.

Em relação aos ferimentos cardíacos, as câmaras mais acometidas foram os ventrículos direito e esquerdo, em igual proporção, 30\% das feridas cardíacas para cada. A seguir as lesões mais frequentes foram no pericárdio sem comprometimento do miocárdico, lesões de átrio direito e átrio esquerdo, respectivamente. Esses dados são comparáveis ao estudo de Spencer Netto et al (2000), no qual $48,8 \%$ das lesões cardíacas foram em ventrículo direito, mas a segunda câmara mais acometida foi o átrio direito com $17,31 \%{ }^{3}$.

Dá-se atenção especial às lesões de aorta torácica nas quais a mortalidade foi de $100 \%$. Segundo Spencer Netto et al (2000), houve acometimento de grandes vasos torácicos em $7,7 \%$ de todos os traumas, comparando-se com 7,3\% deste estudo. Comprova-se então a gravidade do trauma de grandes vasos e a frequência desse trauma que apesar de raro é extremamente fatal ${ }^{3}$.
No trabalho de Huh et al (2003) foi relatado que as principais vias de acesso utilizada para o reparo de lesões pulmonares foram as anteriores em $48 \%$, a posterolateral em 25\%, a bitoracotomia anterior em 24\% e a esternotomia em 3\%. Neste estudo, as vias de acesso mais utilizadas foram as toracotomias anteriores em $67 \%$ dos casos, a bitoracotomia em $16 \%$ e a esternotomia em $9,5 \%$. A diferença entre estes dois estudos em relação às incisões torácicas utilizadas pode ser explicada pelo fato de o primeiro trabalho estar relacionando somente lesões pulmonares e pela presença de um especialista na área de cirurgia torácica que indica com maior frequência a toracotomia posterolateral, fato não verificado nos prontos-socorros selecionados por este estudo que não dispõem de cirurgiões torácicos de plantão ${ }^{10}$.

Numa recente revisão sobre os escores no trauma, Sharma et al (2005), fazem uma análise dos escores encontrados na literatura e relataram que o ISS apresenta falhas. Uma das principais é a ausência de quantificação de múltiplos ferimentos em uma região do corpo. Outra limitação citada é a omissão da probabilidade de morte nos traumatismos cranianos. Apesar destas ponderações, Bergeron (2005) utilizou o ISS para classificar os pacientes em níveis de severidade do trauma ${ }^{6,11,12}$

O ISS foi utilizado neste trabalho por conta da ausência de dados encontrados nos prontuários dos pacientes, que usualmente não apresentavam registros das condições fisiológicas e somente os locais da lesão traumática. Os dados coletados foram estatisticamente significativos $(p=0,004)$ quando comparados com o prognóstico (óbito e cura). Os casos de óbito no paciente de leve gravidade, em grande parte, devem-se aos traumas penetrantes diretos em região precordial, no qual, apesar de um baixo escore no ISS, possuem alta letalidade ${ }^{13,14}$.

O estudo de Huder-Wagner et al (2007) encontrou diferenças significativas na mortalidade dos pacientes, baseado no número de concentrados de hemácias transfundidas $(p=0,001)$, sendo associado principalmente a falência circulatória, em até $53,9 \%$ dos casos. ${ }^{15}$ No presente estudo, os pacientes que receberam mais de três unidades de hemácias tiveram uma maior tendência em evoluir para o óbito comparados aos demais pacientes $(p=0,09)$.

O artigo de Huh et al(2003) relatou uma sobrevida de $26,7 \%$ dos pacientes submetidos à toracotomia para tratamento de lesão do parênquima pulmonar. A taxa de mortalidade nesses pacientes foi de $20,6 \%$, que corresponde à literatura médica, citada em que os pacientes instáveis podem ter uma mortalidade variando em até 30 a 40\% 2,10,16.

Em conclusão, o perfil do paciente vítima de traumatismo torácico submetido à toracotomia exploradora é o seguinte: jovem, do sexo masculino e agredido por arma branca. A toracotomia revelou-se um método de tratamento eficaz com a recuperação em 79,4\% dos casos. Os fatores que mais contribuíram para o êxito letal foram o escore anatômico superior a 14 pontos e a associação com lesões vasculares importantes, como da artéria aorta e veia cava. 
Objective: To analyze patients who underwent thoracotomy for the treatment of chest trauma in the City of Manaus. Methods: We performed a retrospective study through analyzed records in the two main reference hospitals for trauma adults in this city during a period of 5 years. We considered for this study the epidemiological data, causal agent, type of incision, anatomical classification score of trauma, prognostic factors and mortality. Results: We analyzed 124 patients who underwent thoracotomy with a mean age of 28 years. Stab wounds were the most common casual agent (68\%). The main indication for thoracotomy was massive hemothorax with $50.7 \%$ of cases, followed by cardiogenic or hypovolemic shock with $48.4 \%$. There were 28 deaths (20.6\%). Patients with vena cava injuries (5 patients) and aorta lesions (2 patients) had 100\% mortality rate. There was a higher mortality in patients with major index of trauma $(p=0004)$, and largest quantity of blood transfused $(p=0090)$. Conclusion: Thoracic trauma patients submitted to exploratory thoracotomy were young, males and victims of stab wound trauma. The most contributing death factors were the lethal anatomical score, more than 15 points, and the association with major vascular lesions, as the aorta and vena cava.

Key words: Thoracic injuries. Thoracotomy. Trauma severity indices.

\section{REFERENCIAS}

1. Birolini D. Cirurgia de emergência. 1. ${ }^{a}$ ed. São Paulo: Atheneu; 1998.

2. Saad Jr R, Rasslan S. Trauma de tórax em geral. In: Freire E, editor. Trauma: a doença do século. 1. a ed. São Paulo: Atheneu; 2001.

3. Spencer Netto FAC, Campos JM, Lima LFC, Rivera MACP, Kreimer $F$, Silveira RK. Fatores prognósticos de mortalidade em pacientes com trauma cardíaco que chegam à sala de cirurgia. Rev Col Bras Cir. 2000; 28(2):87-94.

4. Solis Chávez SL, Borges Sandrino RB, Fernandez Hernández M Mendes Catasús M. Toracotomías de urgencia y emergencia. Rev Cubana Cir. 2000; 39(1):47-51.

5. Valenzuela DM, Cancino AP, Cabezas FS, Donoso GD, La Torre I. Experiencia en traumatismo torácico: Hospital Valparaíso. Rev Chil Cir.2003; 55(5):449-53.

6. Bergeron E, Lavoie A, Razek T, Belcaid A, Lessard J, Clas D. Penetrating thoracoabdominal injuries in Quebec: implications for surgical training and maintenance of competence. Can J Surg. 2005; 48(4):284-8

7. Lima IS, Lima THB, Patriota SM, Pitta GBB, Bastos AL. Trauma torácico na unidade de emergência. ECML. 1986; 4(1):4-9.

8. Cuba RMB, Bezerra JAF. Traumatismo torácico: estudo retrospectivo de 168 casos. Rev Col Bras Cir. 2005; 32(2):57-9.

9. Fontelles, M. J.; Mantovani, M. Trauma Torácico: fatores de risco de complicações pleuropulmonares pós-drenagem pleural fechada. Rev Col Bras Cir. 2000; 27(6):400-7.

10. Huh J, Wall Jr MJ, Estrera AL, Soltero ER, Mattox KL. Surgical management of traumatic pulmonary injury. Am J Surg. 2003; 186(6):620-4

11. Sharma BR. The injury scale - a valuable tool for forensic documentation of trauma. J Clin Forensic Med. 2005; 12(1):21-8.
12. Demirhan R, Onan B, Oz K, Halezeroglu S. Comprehensive analysis of 4205 patients with chest trauma: a 10-year experience. Interact Cardiovasc Thorac Surg [serial on the internet]. 2009 jun [cited 2009 jun 29]; 1(1): [4 telas]. Available from: http:// icvts.ctsnetjournals.org/cgi/rapidpdf/icvts.2009.206599v1.

13. Baker SP, O'Neill B, Haddon Jr W, Long WB. The injury severity score: a method for describing patients with multiple injuries and evaluating emergency care. J Trauma. 1974; 14(3):187-96.

14. Copes WS, Champion HR, Sacco WJ, Lawnick MM, Keast SL, Bain LW. The injury severity score revised. J Trauma. 1988; 28(1):69-77.

15. Huber-Wagner S, Qvick M, Mussack T, Euler E, Kay MV, MutschlerW, Kanz KG et al. Massive blood transfusion and outcome in 1062 polytrauma patients: a prospective study based on the Trauma Registry of the German Trauma Society. Vox Sang. 2007; 92(1):69-78

16. Van Natta TL, Smith BR, Bricker SD, Putnam BA. Hilar control in penetrating chest trauma: a simplified approach to an underutilized maneuver. J Trauma. 2009; 66(6):1564-9.

Recebido em 24/11/2008

Aceito para publicação em 23/01/2009

Conflito de interesse: nenhum

Fonte de Financiamento: nenhuma

\section{Como citar este artigo:}

Westphal FL, Lima LC, Lima Neto JC, Silva JS, Santos Júnior VL, Westphal DC. Trauma torácico: análise de 124 pacientes submetidos à toracotomia em Manaus. Rev Col Bras Cir. [periódico na Internet] 2009; 36(6). Disponível em URL: http://www.scielo.br/rcbc

\section{Endereço para correspondência:}

Fernando Luiz Westphal

E-mail: f.l.westphal@uol.com.br 